\title{
Clinical performance of stem cell therapy in patients with acute-on-chronic liver failure: a systematic review and meta-analysis
}

\author{
Ran Xue, Qinghua Meng* (i), Jinling Dong, Juan Li, Qinwei Yao, Yueke Zhu and Hongwei Yu
}

\begin{abstract}
Background: Stem cell therapy has been applied in the treatment of acute-on-chronic liver failure (ACLF). However, its clinical efficiency is still debatable. The aim of this systematic review and meta-analysis is to evaluate the clinical efficiency of stem cell therapy in the treatment of ACLF.

Methods: The Cochrane Library, OVID, EMBASE, and PUBMED were searched to December 2017. Both randomized and non-randomized studies, assessing stem cell therapy in patients with ACLF, were included. The outcome measures were total bilirubin (TBIL), alanine transaminase (ALT), international normalized ratio (INR), albumin (ALB), and the model for end-stage liver disease (MELD) score. The quality of evidence was assessed by GRADEpro.

Results: Four randomized controlled trials and six non-randomized controlled trials were included. The TBIL levels significantly decreased at 1-, 3-, 12-month after the stem cell therapy $(p=0.0008 ; p=0.04 ; p=0.007)$. The ALT levels decreased significantly compared with the control group in the short-term $(p<0.00001)$. There was no obvious change in the INR level compared with the control groups $(p=0.64)$. The ALB levels increased markedly as compared with the control groups $(p<0.0001)$. The significant difference can be found in MELD score between stem cell therapy and control groups $(p=0.008)$. Further subgroup analysis for 3 -month clinical performance according to the stem cell types have also been performed.
\end{abstract}

Conclusion: This study suggests that the clinical outcomes of stem cell therapy were satisfied in patients with ACLF in the short-term. MSCs may be better than BM-MNCs in the stem cells transplantation of ACLF. However, more attention should focus on clinical trials in large-volume centers.

Keywords: Stem cell therapy, Acute-on-chronic liver failure, Clinical performance

\section{Background}

Acute-on-chronic liver failure (ACLF) is a global medical problem, with high prevalence and being serious, life-threatening in risk populations [1,2]. ACLF occurs in patients with undiagnosed or previously diagnosed chronic liver disease (CLD) and was distinguished from acute hepatic damages such as coagulopathy and jaundice and complicated within 4 weeks because of sites and/or encephalopathy [3]. ACLF is usually accompanied

*Correspondence: meng_qh@126.com

Department of Critical Care Medicine of Liver Disease, Beijing You-An

Hospital, Capital Medical University, No. 8, Xi tou tiao, You an men wai

Street, Feng tai District, Beijing 100069, People's Republic of China by multiple organ failure, rapid progression and low survival rate. Liver transplantation is the sole solution that has proven beneficial, but the rapid disease progression and lack of donors limit its application [4]. Therefore, it is urgent to find a safe and effective therapeutic approach to ACLF.

Nowadays, a novel therapeutic strategy, stem cell therapy, is beginning to apply in the treatment of ACLF. Stem cells are a kind of undifferentiated cells, which capable of unlimited proliferation [5]. Cells such as hematopoietic stem cells (HSCs), mesenchymal stem cells (MSCs) and unsorted bone marrow cells (BMCs) have all been used in the stem cell therapy for liver diseases. 
Several clinical researches have studied the consequences of stem cell therapy in patients with ACLF [6-8]. Ranged from early conceptual studies [9] to larger random controlled trials (RCTs), the results of those studies showed that stem cell therapy is safe and has beneficial effects on ACLF. However, these studies have several deficiencies such as the small size of the samples and the lacking of the control groups. Meanwhile, some studies have provided conflicting results about the effect of stem cell therapy $[5,10]$. Therefore, it is still debatable for the effect of stem cell therapy in the treatment of ACLF. The purpose of this review is to evaluate the clinical efficiency of stem cell therapy in the treatment of patients with ACLF.

\section{Methods}

This meta-analysis was adhered to the guidelines of Preferred Reporting Items for Systematic Reviews and MetaAnalyses (PRISMA).

\section{Literature search strategies and selection}

We performed our data selection through four databases (PUBMED, EMBASE, Cochrane Library, and OVID) to Dec. 2017. The following medical subject heading $(\mathrm{MeSH})$ terms were used: "hematopoietic stem cells", "mesenchymal stromal cells", "bone marrow", "stem cells", or "stem cell therapy"; "end stage liver disease", "liver cirrhosis", or "acute-on-chronic liver failure". All literatures used in this study were published on humans and in English.

Two reviewers checked the abstract and title of publication independently. If the two reviewers identified the information relevant, we acquired the full text for a total review. Should any disagreements exist, we will discuss it with a third reviewer until there is a final conclusion.

\section{Data extraction and conversion Data extraction}

The picked articles were managed through EndNote X7 (Thomson Reuters, USA). Two independent investigators assessed the qualification of studies by the sequence of title, abstract, and full text. The following data were recorded from the articles: title, first author, journal, year of publication, characteristics of patients (i.e. age, sex), timing of detection (baseline, ongoing/post treatment), and accuracy of in vitro test (sensitivity and specificity).

\section{Inclusion criteria and outcome assessment}

The inclusion criteria were as bellows: (1) specific reporting the indications for stem cell therapy; (2) compared one of the following outcomes at least: alanine transaminase (ALT), total bilirubin (TBIL), albumin (ALB), international normalized ratio (INR), model for endstage liver disease (MELD) score.

\section{Exclusion criteria}

The following trials were excluded: (1) it was difficult to extract or reasonably evaluate the data from the reliably articles; (2) those without clear outcomes; (3) abstracts, expert opinions, editorials, case reports, letters, and studies without control groups.

\section{Statistical analysis}

The quality of evidence was assessed by GRADEpro $[11,12]$. It is preferred that the data showed as changes. When changes were not available, but could be managed through the calculation of the data, they will be calculated through appropriate methods [13]. We performed the Meta-analysis by Review Manager (version 5.3). We use a random-effects model to evaluate the heterogeneity and $\mathrm{I}^{2}>50 \%$ with $\mathrm{p}<0.10$ was statistically significant. The analysis of continuous variables was preceded by the mean difference (MD) as the summary statistic through the Inverse-Variance method, and the result was shown with a $95 \%$ confidential interval (CI). It was considered to be significant when $\mathrm{p}<0.05$.

\section{Results}

\section{Literature search and characteristics}

A total of 541 potentially eligible studies were identified and reviewed. 458 of records, of which titles or abstracts were screened, were excluded. 79 studies remained were evaluated in detail. And 69 of these studies were excluded, 7 of which with no comparative studies, 10 of which were reviews and meta-analysis, 23 of which were excluded for undesired treatment or unexpected inclusion of participants, 12 of which were involved patients not only ACLF, 15 of which did not report the outcome interested, and 2 of which were unpublished by English.

As a result, 10 of the records were included in this meta-analysis, including five RCTs, five non-randomized controlled trial. A detailed flow chart of the study selection and the literature search is shown in Fig. 1. Although the authors were contacted, we were unable to obtain additional information to include in the analysis. The characteristics of the included studies are presented in Table 1.

\section{Stem cell therapy improved liver function and reduced liver injury}

In advanced liver disease, the level of the serum ALB, which is a major protein produced by the liver, is reduced. INR reflects the coagulation function. The liver 

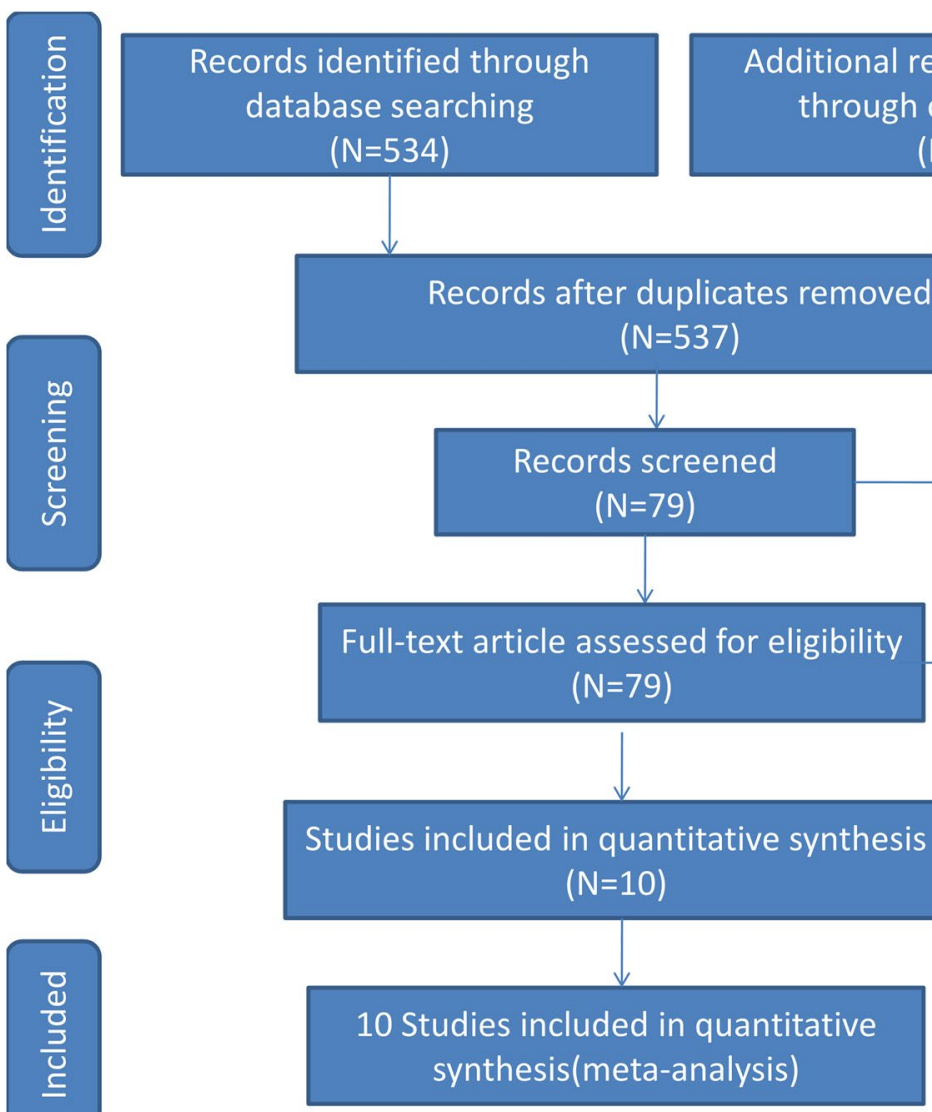

Additional records identified

through other source $(\mathrm{N}=7)$

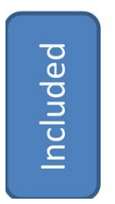

Records excluded

$(N=458)$

- Studies excluded for

unexpected inclusion of

participants or undesired

treatment $(n=23)$

with no comparative

studies(n=7)

- Involved patients not only

ACLF $(n=12)$

-Review and meta-analysis

$(n=10)$

-Studies did not report the

outcome interested $(n=15)$

- Unpublished by English ( $\mathrm{n}=2$ )

Fig. 1 Flow diagram of the article selection process

is the place where most of coagulation factors and variety of anti-thrombin synthesis were produced [2]. Advanced liver disease can significantly reduce the synthesis of coagulation factors, and affect the coagulation function.

We analyzed the ALB and INR levels to investigate the impact of stem cell therapy on liver synthesis functions. Compared with the control groups, the ALB levels increased dramatically after the stem cell therapy $(p<0.0001)$. But heterogeneity should be considered about ALB level $\left(\mathrm{p}<0.00001\right.$; with $\left.\mathrm{I}^{2}=87 \%\right)$. The subgroup analysis showed that the ALB levels increased significantly 9, 12, 24 months after the stem cell therapy (Fig. 2).

The INR levels didn't show any significant change compared with the control groups $(p=0.64)$. However, heterogeneity was especially high among these studies, although subgroup analysis was done $\left(\mathrm{I}^{2}: 82-95 \%\right)$ (Fig. 3).

ALT is an enzyme in liver cells; it leaks into the general circulation when liver cells are damaged. It is considered to be a particular indicator of liver inflammation. The TBIL level can increase in biliary tract or liver diseases.
In this research, the effects of the stem cell therapy on reducing liver damage were also evaluated through analyzing TBIL and ALT levels. The TBIL level significantly decreased 1, 3, 12 month after the stem cell therapy $(p=0.0008 ; p=0.04 ; p=0.007)$. Compared with the control group, no significant difference was found at 1 week, 6, 9 and 24 months time points (Fig. 4). No heterogeneity was detected regarding the TBIL level, except for 3, 6 and 9 months. But it could be explained by only one or two studies.

Compared with the control group, the ALT levels decreased significantly after stem cell therapy $(\mathrm{p}<0.00001)$ (Fig. 5). The subgroup analysis showed that the ALT level in the stem cell therapy group also decreased, and was lower than baseline and the corresponding control group at the 1 week, 1 and 6 months time points. There were no significant differences in ALT level at 9, 12 and 24 months after stem cell therapy. No heterogeneity was found regarding the ALT level at 1 week, 6 and 9 months. However, heterogeneity should be taken into consideration for the ALT level at 1, 3 and 


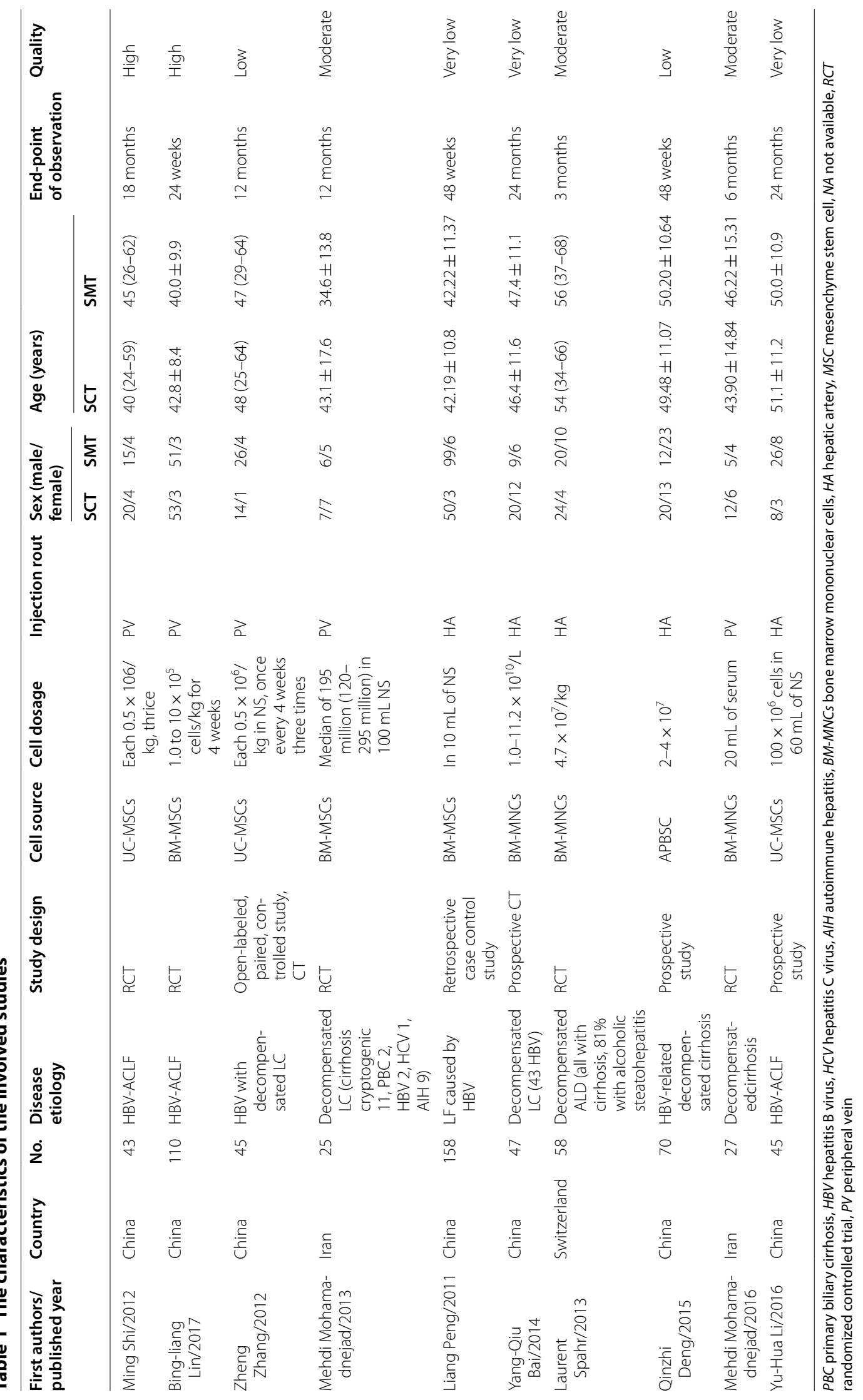




\begin{tabular}{|c|c|c|c|c|c|c|c|}
\hline \multirow{2}{*}{ Study or Subgroup } & \multicolumn{3}{|c|}{ Experimental } & \multicolumn{2}{|c|}{ Control } & & \\
\hline & Mean & SD & Total & \multicolumn{2}{|c|}{ Control } & Total & \\
\hline \multicolumn{8}{|l|}{ 2.1.1 Basline } \\
\hline Bing-liang Lin,2017 & 35.9 & 4.3 & 56 & 34.7 & 4.4 & 54 & \\
\hline Liang Peng, 2011 & 29.67 & 3.14 & 53 & 29.4 & 3.92 & 105 & \\
\hline Mehdi Mohamadnejad, 2013 & 3.3 & 0.6 & 14 & 3.5 & 0.6 & 11 & \\
\hline MEHDI MOHAMADNEJAD, 2016 & 3.16 & 0.52 & 10 & 3.71 & 0.33 & 9 & \\
\hline MING SHI,2012 & 31.4 & 2.7 & 24 & 28.2 & 3.9 & 19 & \\
\hline Qinzhi Deng, 2015 & 30.1 & 3.75 & 33 & 28.75 & 8.2 & 35 & \\
\hline Yang-Qiu Bai, 2014 & 27.9 & 2.5 & 32 & 28.1 & 2.8 & 15 & \\
\hline Yu-Hua Li, 2016 & 26 & 4.7 & 11 & 28.1 & 6.2 & 34 & \\
\hline Zheng Zhang,2012 & 28.5 & 5.1 & 30 & 28 & 4.9 & 15 & \\
\hline Subtotal $(95 \% \mathrm{Cl})$ & & & 263 & & & 297 & \\
\hline \multicolumn{8}{|c|}{$\begin{array}{l}\text { Heterogeneity: } \text { Tau }^{2}=0.09 ; \mathrm{Ch}^{2}=17.50, \text { df }=8(P=0.03) ; 1^{2}=54 \% \\
\text { Test for overall effect: } Z=0.40(P=0.69)\end{array}$} \\
\hline \multicolumn{8}{|l|}{ 2.1.2 1 week } \\
\hline Bing-liang Lin,2017 & 38.1 & 1.75 & 56 & 34.7 & 1.5 & 54 & \\
\hline Liang Peng, 2011 & 32.33 & 3.5 & 53 & 31.93 & 3.26 & 105 & \\
\hline MING SHI,2012 & 26.1 & 3.1 & 24 & 25.8 & 2.1 & 19 & \\
\hline Yang-Qiu Bai, 2014 & 28.4 & 2.7 & 32 & 29.2 & 2.5 & 15 & \\
\hline Zheng Zhang,2012 & 30.5 & 3.1 & 30 & 29.6 & 4.2 & 15 & \\
\hline Subtotal $(95 \% \mathrm{Cl})$ & & & 195 & & & 208 & \\
\hline \multirow{2}{*}{\multicolumn{8}{|c|}{$\begin{array}{l}\text { Heterogeneity: } \mathrm{Tau}^{2}=0.87 ; \mathrm{Ch}^{2}=57.28, \mathrm{df}=4(P<0.00001) ; \mathrm{I}^{2}=93 \% \\
\text { Test for overall effect: } Z=1.06(P=0.29)\end{array}$}} \\
\hline & & & & & & & \\
\hline \multicolumn{8}{|l|}{ 2.1.31 month } \\
\hline Bing-liang Lin,2017 & 38.6 & 1.75 & 56 & 38.7 & 1.5 & 54 & \\
\hline Liang Peng, 2011 & 35.92 & 1.11 & 53 & 34.47 & 1.46 & 105 & \\
\hline MING SHI,2012 & 28.6 & 5.4 & 24 & 26.5 & 2.4 & 19 & \\
\hline Yang-Qiu Bai, 2014 & 30.2 & 3.2 & 32 & 29.8 & 2.8 & 15 & \\
\hline Yu-Hua Li, 2016 & 31.3 & 2.1 & 11 & 28.3 & 3 & 34 & \\
\hline Zheng Zhang,2012 & 31.4 & 5 & 30 & 33 & 7 & 15 & \\
\hline Subtotal $(95 \% \mathrm{Cl})$ & & & 206 & & & 242 & \\
\hline \multirow{2}{*}{\multicolumn{8}{|c|}{$\begin{array}{l}\text { Heterogeneity: } \text { Tau }^{2}=0.31 ; \mathrm{Chi}^{2}=27.93, \text { df }=5(P<0.0001) ;\left.\right|^{2}=82 \% \\
\text { Test for overall effect: } Z=1.56(P=0.12)\end{array}$}} \\
\hline & & & & & & & \\
\hline \multicolumn{8}{|l|}{ 2.1.4 3 months } \\
\hline Bing-liang Lin,2017 & 37.2 & 1.125 & 56 & 38.5 & 1.5 & 54 & \\
\hline Liang Peng, 2011 & 36.75 & 2.27 & 53 & 33.93 & 1.98 & 105 & \\
\hline Mehdi Mohamadnejad, 2013 & 3.3 & 0.7 & 14 & 3.8 & 0.5 & 11 & \\
\hline MEHDI MOHAMADNEJAD, 2016 & 3.26 & 0.85 & 10 & 3.12 & 0.71 & 9 & \\
\hline MING SHI,2012 & 34.7 & 7 & 24 & 28.3 & 0.5 & 19 & \\
\hline Qinzhi Deng, 2015 & 31.46 & 4.23 & 3 & 29.11 & 6.19 & 35 & \\
\hline Yang-Qiu Bai, 2014 & 33.5 & 3.6 & 32 & 30.3 & 2.2 & 15 & \\
\hline Yu-Hua Li, 2016 & 34 & 3 & 11 & 31.7 & 3.2 & 34 & \\
\hline Zheng Zhang,2012 & 32.5 & 5.5 & 30 & 30 & 5 & 15 & \\
\hline 0 & & & 233 & & & 297 & \\
\hline
\end{tabular}

Std. Mean Difference

Std. Mean Difference

2.1.1 Basline

IV. Random. $95 \% \mathrm{C}$

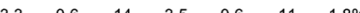

$-0.32[-1.12,0.47]$

$-1.19[-2.19,-0.20]$
$0.96[0.32,1.60]$

$0.21[-0.27,0.68]$

$-0.08[-0.69,0.54]$

$-0.35[-1.03,0.33]$

$0.10[-0.52,0.72]$
$\mathbf{0 . 0 6}[-0.22,0.33]$

Zheng Zhang,201

2.1.2 1 week

Lin, 2017

$2.07[1.60,2.53]$

$0.12[-0.21,0.45]$

$0.11[-0.49,0.71]$

$-0.30[-0.91,0.32]$

$0.25[-0.37,0.88]$

$0.46[-0.39,1.31]$

Test for overall effect: $Z=1.06(P=0.29)$

2.1.31 month

$-0.06[-0.43,0.31]$

$1.07[0.72,1.42]$

$0.47[-0.14,1.09]$

$0.13[-0.49,0.74]$

$1.05[0.33,1.76]$

$-0.27[-0.90,0.35]$
$\mathbf{0 . 4 0}[-0.10,0.89]$

Heterogeneity: $\mathrm{Tau}^{2}=0.97 ; \mathrm{Chi}^{2}=90.14, \mathrm{df}=8(\mathrm{P}<0.00001) ; \mathrm{I}^{2}=91 \%$

Test for overall effect: $Z=1.13(P=0.26)$

2.1.5 6 months

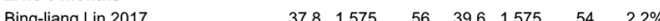

$\begin{array}{lrrrrrrr}\text { Liang Peng, 2011 } & 36.93 & 2.43 & 53 & 34.33 & 2.61 & 105 & 2.2 \%\end{array}$

$\begin{array}{lrrrrrrr}\text { Mehdi Mohamadnejad, } 2013 & 3.3 & 0.5 & 14 & 3.9 & 0.7 & 11 & 1.7 \%\end{array}$

$\begin{array}{lrrrrrrr}\text { MEHDI MOHAMADNEJAD, } 2016 & 3.3 & 0.81 & 10 & 3.63 & 0.43 & 9 & 1.6 \%\end{array}$

MING SHI,2012

$\begin{array}{lrllllll}\text { Qinzhi Deng, 2015 } & 32.82 & 5.1 & 33 & 29.16 & 6.13 & 35 & 2.1 \%\end{array}$

$\begin{array}{llllllll}\text { Yang-Qiu Bai, } 2014 & 33.9 & 3.5 & 32 & 30.4 & 3.3 & 15 & 1.9 \%\end{array}$

$\begin{array}{llllllll}\text { Yu-Hua Li, } 2016 & 36.2 & 2.8 & 11 & 34.1 & 3.4 & 34 & 1.9 \%\end{array}$

$\begin{array}{lrrrrrrr}\text { Zheng Zhang, } 2012 & 33 & 4.7 & 30 & 32 & 4.1 & 15 & 2.0 \% \\ \text { Subtotal }(95 \% \mathrm{Cl}) & & & 263 & & & 297 & 17.6 \%\end{array}$

Heterogeneity: $\mathrm{Tau}^{2}=0.84 ; \mathrm{Chi}^{2}=90.68, \mathrm{df}=8(\mathrm{P}<0.00001) ; \mathrm{I}^{2}=91 \%$

Test for overall effect: $Z=0.77(P=0.44)$

2.1.6 9 months

Liang Peng, 2011

MING SHI,2012

Qinzhi Deng, 2015

Qinzhi Deng, 2015

Sheng Zhang,2012

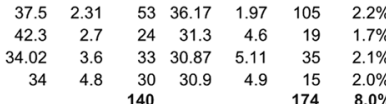

Heterogeneity: $\mathrm{Tau}^{2}=0.51 ; \mathrm{Ch}^{2}=23.82, \mathrm{df}=3(P<0.0001) ; \mathrm{l}^{2}=87 \%$

Test for overall effect: $Z=2.93(P=0.003)$

\subsubsection{2 months}

$\begin{array}{llllllll}\text { Liang Peng, } 2011 & 36.83 & 2.18 & 53 & 36.73 & 2.71 & 105 & 2.2 \%\end{array}$

$\begin{array}{lrrrrrrr}\text { Mendi Monamadnejad, } 2013 & 3.1 & 0.8 & 14 & 3.9 & 0.3 & 11 & 1.7 \%\end{array}$

MING SHI2012

Qinzhi Deng, 2015

Yang-Qiu Bai, 2014

Yu-Hua Li, 2016

Zheng Zhang, 2012

ubtotal $(95 \% \mathrm{Cl})$

$\begin{array}{rrrrrrr}3.1 & 0.8 & 14 & 3.9 & 0.3 & 11 & 1.7 \% \\ 41.8 & 5.3 & 24 & 30.8 & 4 & 19 & 1.8 \%\end{array}$

$\begin{array}{rrrrrrr}41.8 & 5.3 & 24 & 30.8 & 4 & 19 & 1.8 \% \\ 34.7 & 3.8 & 33 & 30.58 & 5.43 & 35 & 2.1 \%\end{array}$

$\begin{array}{rrrrrrr}34.7 & 3.8 & 33 & 30.58 & 5.43 & 35 & 2.1 \% \\ 34.5 & 4.2 & 32 & 29.7 & 4.3 & 15 & 1.9 \%\end{array}$

$\begin{array}{llllrrr}37.3 & 2.4 & 11 & 33.5 & 4 & 34 & 1.9 \%\end{array}$

$\begin{array}{llll}434 & 197 & 13.6 \%\end{array}$

Test for overall effect: $Z=2.15(P=0.03)$

2.1.8 24 months

Yang-Qiu Bai, 2014

Subtotal $(95 \% \mathrm{Cl})$

$-0.98[-1.37,-0.58]$

$1.35[0.99,1.71]$

$-0.78[-1.60,0.05]$

$0.17[-0.73,1.07]$

$1.20[0.54,1.85]$

$0.38[-0.81,1.56]$

$0.72[0.02,1.41]$

$0.72[0.02,1.41]$

$0.46[-0.17,1.09]$

$-1.13[-1.54,-0.73]$

$1.01[0.67,1.36]$

$-0.97[-1.82,-0.13]$

$-0.48[-1.39,0.44]$

$1.21[0.55,1.86]$

$0.64[0.15,1.13]$

$1.00[0.35,1.65]$

$0.63[-0.06,1.32]$

$0.22[-0.40,0.84]$

$0.25[-0.38,0.89]$

$0.63[0.30,0.97]$

$2.95[2.06,3.84]$

$0.70[0.21,1.19]$

$0.63[-0.00,1.26]$

$1.14[0.38,1.90]$

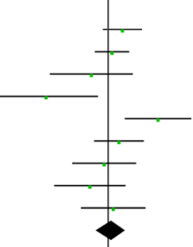

$0.04[-0.29,0.37]$ $-1.22[-2.09,-0.35]$ $2.26[1.48,3.05]$

$0.86[0.37,1.36]$

$1.12[0.46,1.77]$

$1.01[0.30,1.73]$

$0.90[0.25,1.55]$

$0.71[0.06,1.37]$

Total $(95 \% \mathrm{Cl})$

1540

$1798 \quad 100.0 \%$

Heterogeneity: $\mathrm{Tau}^{2}=0.50 ; \mathrm{Chi}^{2}=389.27, \mathrm{df}=50(\mathrm{P}<0.00001) ; \mathrm{I}^{2}=87 \%$

Test for overall effect: $Z=3.93(P<0.0001)$

Test for o f

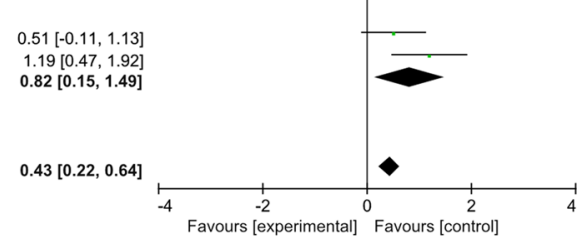

Fig. 2 Forest plot of the ALB level during follow up 


\begin{tabular}{|c|c|c|c|c|c|c|c|c|c|c|c|}
\hline \multirow{3}{*}{$\begin{array}{l}\text { Study or Subgroup } \\
3.1 .1 \text { Basline }\end{array}$} & \multicolumn{3}{|c|}{ Experimental } & \multicolumn{2}{|c|}{ Control } & & & \multirow{3}{*}{$\begin{array}{l}\text { Mean Difference } \\
\text { IV. Random, } 95 \% \mathrm{CI}\end{array}$} & \multicolumn{3}{|c|}{ Mean Difference } \\
\hline & \multirow[t]{2}{*}{ Mean } & \multicolumn{2}{|c|}{ SD Total } & \multirow[t]{2}{*}{ Mean } & \multirow[t]{2}{*}{ SD } & \multirow{2}{*}{\multicolumn{2}{|c|}{ Total Weight }} & & \multicolumn{3}{|c|}{ IV, Random, $95 \% \mathrm{Cl}$} \\
\hline & & & & & & & & & & & \\
\hline Bing-liang Lin,2017 & 2.2 & 0.225 & 56 & 2.4 & 0.225 & 54 & $4.7 \%$ & $-0.20[-0.28,-0.12]$ & - & & \\
\hline Mehdi Mohamadnejad, 2013 & 1.5 & 0.5 & 14 & 1.6 & 0.2 & 11 & $2.3 \%$ & $-0.10[-0.39,0.19]$ & & - & \\
\hline MEHDI MOHAMADNEJAD, 2016 & 1.57 & 0.18 & 10 & 1.32 & 0.34 & 9 & $2.7 \%$ & $0.25[0.00,0.50]$ & & & \\
\hline Yu-Hua Li, 2016 & 2.14 & 0.26 & 11 & 2.07 & 0.15 & 34 & $3.7 \%$ & $0.07[-0.09,0.23]$ & & & \\
\hline Zheng Zhang,2012 & 1.4 & 0.3 & 30 & 1.3 & 0.15 & 15 & $4.1 \%$ & $0.10[-0.03,0.23]$ & & & \\
\hline Subtotal $(95 \% \mathrm{Cl})$ & & & 121 & & & 123 & $17.5 \%$ & $0.02[-0.16,0.19]$ & & & \\
\hline \multicolumn{12}{|c|}{$\begin{array}{l}\text { Heterogeneity: } \text { Tau }^{2}=0.03 ; \mathrm{Chi}^{2}=24.85, \mathrm{df}=4(P<0.0001) ; \mathrm{I}^{2}=84 \% \\
\text { Test for overall effect: } Z=0.18(P=0.86)\end{array}$} \\
\hline \multicolumn{12}{|l|}{ 3.1.2 1 week } \\
\hline Bing-liang Lin, 2017 & 2.2 & 0.225 & 56 & 2.5 & 0.3 & 54 & $4.5 \%$ & $-0.30[-0.40,-0.20]$ & & & \\
\hline Zheng Zhang,2012 & 1.32 & 0.35 & 30 & 1.27 & 0.2 & 15 & $3.7 \%$ & $0.05[-0.11,0.21]$ & & & \\
\hline Subtotal $(95 \% \mathrm{Cl})$ & & & 86 & & & 69 & $8.2 \%$ & $-0.13[-0.47,0.21]$ & & & \\
\hline \multicolumn{11}{|c|}{$\begin{array}{l}\text { Heterogeneity: } \text { Tau }^{2}=0.06 ; \mathrm{Chi}^{2}=13.14, \mathrm{df}=1(P=0.0003) ; \mathrm{I}^{2}=92 \% \\
\text { Test for overall effect: } Z=0.75(P=0.45)\end{array}$} & \\
\hline \multicolumn{12}{|l|}{ 3.1.3 1 month } \\
\hline Bing-liang Lin,2017 & 2.2 & 0.25 & 56 & 2.1 & 0.35 & 54 & $4.3 \%$ & $0.10[-0.01,0.21]$ & & $=$ & \\
\hline Yu-Hua Li, 2016 & 2.01 & 0.12 & 11 & 2.17 & 0.27 & 34 & $4.3 \%$ & $-0.16[-0.28,-0.04]$ & & & \\
\hline Zheng Zhang,2012 & 1.3 & 0.32 & 30 & 1.2 & 0.3 & 15 & $3.4 \%$ & $0.10[-0.09,0.29]$ & & & \\
\hline Subtotal $(95 \% \mathrm{Cl})$ & & & 97 & & & 103 & $12.0 \%$ & $0.01[-0.18,0.19]$ & & & \\
\hline $\begin{array}{l}\text { Heterogeneity: } \mathrm{Tau}^{2}=0.02 ; \mathrm{Ch}^{2}= \\
\text { Test for overall effect: } Z=0.08 \text { (P }\end{array}$ & $\begin{array}{l}11.38, \mathrm{df} \\
0.94)\end{array}$ & $f=2(P=$ & $0.003)$ & $; 1^{2}=8$ & & & & & & & \\
\hline 3.1.4 3 months & & & & & & & & & & & \\
\hline Bing-liang Lin,2017 & 1.8 & 0.225 & 56 & 1.7 & 0.15 & 54 & $4.8 \%$ & $0.10[0.03,0.17]$ & & - & \\
\hline Mehdi Mohamadnejad, 2013 & 1.8 & 0.5 & 14 & 1.6 & 0.4 & 11 & $1.8 \%$ & $0.20[-0.15,0.55]$ & & & \\
\hline MEHDI MOHAMADNEJAD, 2016 & 1.54 & 0.35 & 10 & 1.48 & 0.34 & 9 & $2.1 \%$ & $0.06[-0.25,0.37]$ & & & \\
\hline Yu-Hua Li, 2016 & 1.57 & 0.19 & 11 & 1.83 & 0.2 & 34 & $4.1 \%$ & $-0.26[-0.39,-0.13]$ & 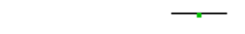 & & \\
\hline Zheng Zhang,2012 & 1.3 & 0.22 & 30 & 1.2 & 0.15 & 15 & $4.4 \%$ & $0.10[-0.01,0.21]$ & & & \\
\hline Subtotal $(95 \% \mathrm{Cl})$ & & & 121 & & & 123 & $17.2 \%$ & $0.02[-0.14,0.18]$ & & & \\
\hline $\begin{array}{l}\text { Heterogeneity: } \mathrm{Tau}^{2}=0.02 ; \mathrm{Ch}^{2}= \\
\text { Test forerall effect: } Z=0.27 \text { (P }\end{array}$ & $\begin{array}{l}24.87, \mathrm{df} \\
0.79)\end{array}$ & $f=4(P$ & 0.000 & 1); $1^{2}=$ & & & & & & & \\
\hline 3.1.5 6 months & & & & & & & & & & & \\
\hline Bing-liang Lin,2017 & 1.4 & 0.15 & 56 & 1.5 & 0.125 & 54 & $5.0 \%$ & $-0.10[-0.15,-0.05]$ & - & & \\
\hline Mehdi Mohamadnejad, 2013 & 1.5 & 0.4 & 14 & 1.4 & 0.3 & 11 & $2.4 \%$ & $0.10[-0.17,0.37]$ & & & \\
\hline MEHDI MOHAMADNEJAD, 2016 & 1.67 & 0.33 & 10 & 1.41 & 0.24 & 9 & $2.6 \%$ & $0.26[0.00,0.52]$ & & & \\
\hline Yu-Hua Li, 2016 & 1.43 & 0.13 & 11 & 1.6 & 0.23 & 34 & $4.4 \%$ & $-0.17[-0.28,-0.06]$ & - & & \\
\hline Zheng Zhang,2012 & 1.3 & 0.2 & 30 & 1.2 & 0.1 & 15 & $4.6 \%$ & $0.10[0.01,0.19]$ & & & \\
\hline Subtotal $(95 \% \mathrm{Cl})$ & & & 121 & & & 123 & $19.0 \%$ & $0.01[-0.13,0.14]$ & & & \\
\hline $\begin{array}{l}\text { Heterogeneity: } \mathrm{Tau}^{2}=0.02 ; \mathrm{Ch}^{2}= \\
\text { Test for overall effect: } Z=0.09 \text { (P }\end{array}$ & $\begin{array}{l}\text { 26.18, df } \\
0.93)\end{array}$ & $f=4(P$ & 0.000 & 1); $1^{2}=$ & $85 \%$ & & & & & & \\
\hline 3.1.6 9 months & & & & & & & & & & & \\
\hline $\begin{array}{l}\text { Zheng Zhang,2012 } \\
\text { Subtotal (95\% Cl) }\end{array}$ & 1.28 & 0.1 & $\begin{array}{l}30 \\
30\end{array}$ & 1.25 & 0.12 & $\begin{array}{l}15 \\
15\end{array}$ & $\begin{array}{l}4.8 \% \\
4.8 \%\end{array}$ & $\begin{array}{l}0.03[-0.04,0.10] \\
0.03[-0.04,0.10]\end{array}$ & & & \\
\hline Heterogeneity: Not applicable & & & & & & & & & & & \\
\hline Test for overall effect: $Z=0.83(P$ & $0.40)$ & & & & & & & & & & \\
\hline 3.1.7 12 months & & & & & & & & & & & \\
\hline Mehdi Mohamadnejad, 2013 & 1.5 & 0.3 & 14 & 1.3 & 0.4 & 11 & $2.3 \%$ & $0.20[-0.08,0.48]$ & & & \\
\hline Yu-Hua Li, 2016 & 1.28 & 0.05 & 11 & 1.44 & 0.28 & 34 & $4.5 \%$ & $-0.16[-0.26,-0.06]$ & & & \\
\hline Zheng Zhang,2012 & 1.25 & 0.15 & 30 & 1.2 & 0.12 & 15 & $4.7 \%$ & $0.05[-0.03,0.13]$ & & & \\
\hline Subtotal $(95 \% \mathrm{Cl})$ & & & 55 & & & 60 & $11.6 \%$ & $0.00[-0.18,0.18]$ & & & \\
\hline Heterogeneity: $\mathrm{Tau}^{2}=0.02 ; \mathrm{Ch}^{2}=$ & 12.90, df & $f=2(P=$ & 0.002) & $1^{2}=8$ & & & & & & & \\
\hline Test for overall effect: $Z=0.01(P$ & $0.99)$ & & & & & & & & & & \\
\hline 3.1.8 24 months & & & & & & & & & & & \\
\hline Yu-Hua Li, 2016 & 1.12 & 0.1 & 11 & 1.35 & 0.09 & 34 & $4.9 \%$ & $-0.23[-0.30,-0.16]$ & - & & \\
\hline Zheng Zhang,2012 & 1.2 & 0.13 & 30 & 1.19 & 0.12 & 15 & $4.8 \%$ & $0.01[-0.07,0.09]$ & & & \\
\hline Subtotal $(95 \% \mathrm{Cl})$ & & & 41 & & & 49 & $9.6 \%$ & $-0.11[-0.35,0.12]$ & & & \\
\hline Heterogeneity: $\mathrm{Tau}^{2}=0.03 ; \mathrm{Chi}^{2}=$ & 21.57, df & $f=1(P$ & 0.0000 & 01); $\left.\right|^{2}=$ & $=95 \%$ & & & & & & \\
\hline Test for overall effect: $Z=0.92(P$ & $0.36)$ & & & & & & & & & & \\
\hline Total $(95 \% \mathrm{Cl})$ & & & 672 & & & 665 & $100.0 \%$ & $-0.01[-0.07,0.04]$ & & & \\
\hline $\begin{array}{l}\text { Heterogeneity: } \mathrm{Tau}^{2}=0.02 ; \mathrm{Chi}^{2}= \\
\text { Test for overall effect: } Z=0.46(P \\
\text { Test for subaroun differences: Chi }\end{array}$ & $\begin{array}{l}175.29, \mathrm{~d} \\
0.64) \\
=2.01 . \mathrm{d}\end{array}$ & $\begin{array}{l}\mathrm{df}=25(1 \\
\mathrm{df}=7(\mathrm{P}\end{array}$ & $\begin{array}{l}<0.00 \\
=0.961\end{array}$ & $0001) ;$ & $\begin{array}{l}\left.\right|^{2}=86 \% \\
\%\end{array}$ & & & & $\begin{array}{ll}-1 & -0.5 \\
\text { Favours [experimental] }\end{array}$ & $\begin{array}{ll}0 & 0.5 \\
& \text { Favours [control] }\end{array}$ & 1 \\
\hline Fig. 3 Forest plot of the INR le & el durin & hg follo & v up & & & & & & & & \\
\hline
\end{tabular}




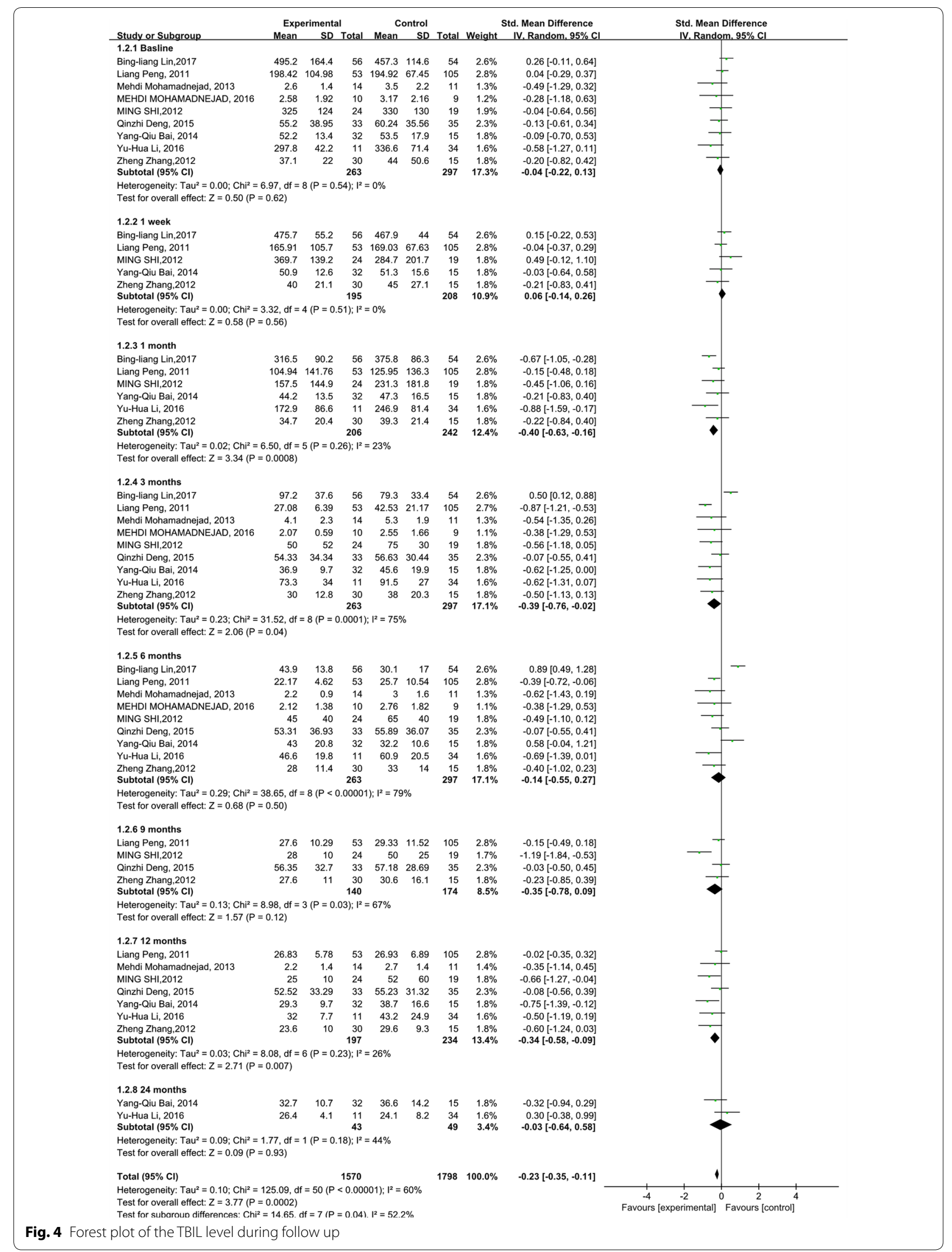




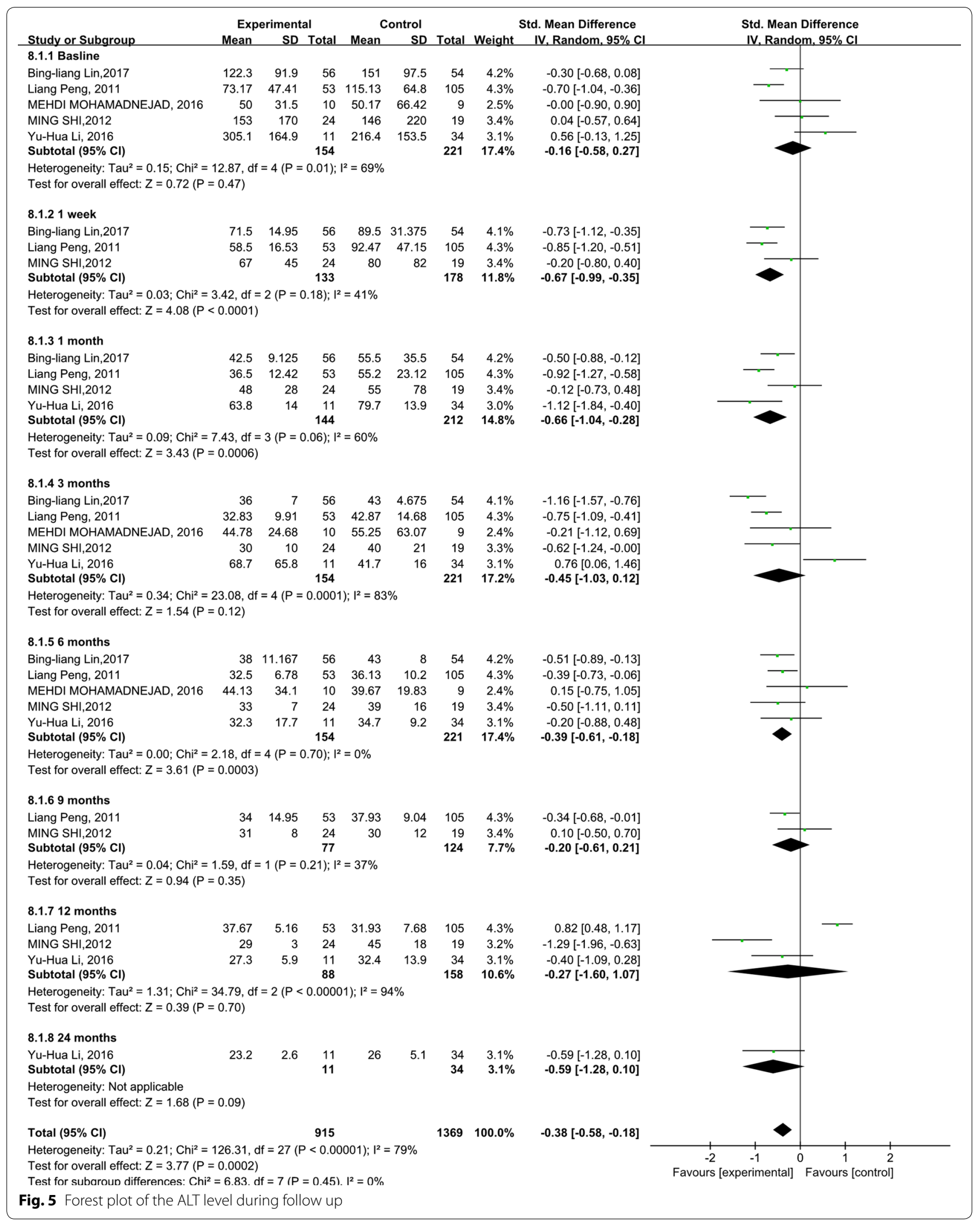


12 months. But it could be explained by only one or two studies.

\section{Stem cell therapy reduced the MELD score in ACLF patients} The MELD score is mostly used to assess the prognosis of end-stage liver diseases [14]. We observed that the MELD scores were both decreased in the stem cell therapy and control groups. Significant differences can be found between the stem cell therapy and control groups $(\mathrm{p}=0.008)$ (Fig. 6). Moreover, heterogeneity was high within these studies, although subgroup analysis was done $\left(\mathrm{I}^{2}\right.$ : $\left.62-100 \%\right)$. But it could be also explained by only one or two studies.

\section{Further subgroup analysis for 3-month clinical performance according to the stem cell types}

Due to the high heterogeneity, we continued to perform subgroup analyses on the effects of stem cell type at 3 months after stem cell therapy (Fig. 7). For ALB, heterogeneity was still obvious in BM-MSCs and BM-MNCs $\left(\mathrm{I}^{2}: 91-97 \%\right)$, not much high in UC-MSCs $\left(\mathrm{I}^{2}=65 \%\right)$. It was shown that no heterogeneity was found for INR in BM-MSCs group $\left(\mathrm{I}^{2}=0 \%\right)$. And INR had a significant change compared with the control groups $(\mathrm{p}=0.004)$. However, it is the meta-analysis result of 2 studies. The credibility of the result is not high. As for TBIL, there were no differences for heterogeneity in UC-MSCs and BM-MNCs $\left(\mathrm{I}^{2}=0 \% ; \mathrm{I}^{2}=56 \%\right)$. It seems that UC-MSCs had a better effect on reducing the TBIL than BM-MNCs $(p=0.01)$. After the subgroup analysis on the effects of stem cell type at 3 months of follow-up for ALT, it was shown that no heterogeneity was found $\left(\mathrm{I}^{2}=20 \%\right)$. Stem cell therapy can significantly reduce liver injury at 3 months of follow-up ( $<<0.00001)$. BM-MSCs can obviously reduce the ALT level, which is better than other stem cell type $(\mathrm{p}<0.00001)$. For MELD score, after subgroup analysis on the effects of stem cell type, there was still a high heterogeneity $\left(\mathrm{I}^{2}=87 \%\right)$, mainly in BM-MSCs group. It is indicated that UC-MSCs can significantly decrease the MELD score, which is better than BMMNCs at 3 months of follow-up ( $<<0.001)$.

\section{Methodological quality of the included studies}

The quality assessment of each study is found in Table 1. The evaluation items were as follows: performance bias, detection bias, selection bias (random sequence generation, allocation concealment), attrition bias, reporting bias and other bias.

\section{Discussion}

ACLF is a serious life-threatening disease with high prevalence. Due to the rapid progression and low survival rate of ACLF, it is urgent to find a safe and effective therapeutic approach to ACLF [1-3]. In this study, we found that stem cell therapy was able to improve liver function and alleviate liver damage. Meanwhile, stem cell therapy can also reduce the MELD score for ACLF patients. These results suggested that stem cell therapy can be considered to be a potential supplementary therapeutic approach to improve liver function in patients with ACLF.

The immunomodulatory and reparative functions of stem cells have been demonstrated to be therapeutically valuable for treating various diseases, including autoimmune diseases, diabetes, myocardial infarction, and organ therapy rejection [15-17]. The present systematic review and meta-analysis underlines the feasibility of stem cell treatment for patients with ACLF. In the process of transplantation, stem cells, especially MSCs, can not only differentiate into corresponding tissue cells, but also have strong immunoregulatory effects in various organs, such as inhibiting the proliferation of $\mathrm{T}$ cells in the recipient, altering the proportion of $\mathrm{T}$ cell subsets and the function of B cells, inhibiting dendritic cell differentiation and NK cell proliferation, reducing the number of inflammatory cytokines, increasing anti-inflammatory cytokines quantity, adjusting immune abnormalities and disorders, and improving the inflammatory environment around the damaged area $[18,19]$. However, regardless of substantial clinical data, which proved the safety of stem cell therapy, there were no considerable supporting reports to confirm the immunological status of patients. As the immune profile of patients is the key to foresee the short and long-term graft clinical outcome, subsequent studies need to focus on monitoring of both clinical as well as immunological parameters [20]. Immunological parameters include T cells, regulatory T cells, B cells, NK cells, dendritic cell and intracellular cytokine expression.

As for the clinical monitoring, this study demonstrated that stem cell therapy improved the liver synthesis functions. ALB levels increased significantly 9, 12, 24 months after the stem cell therapy. But heterogeneity was still high among these studies during the 9, 12 months of follow-up, and could not be explained by only one or two studies. As for the heterogeneity of ALB level data, we considered that ALB transfusion may affect the serum ALB levels during the treatment of ACLF. Meanwhile, there existed heterogeneity at the baseline of ALB level in this study. In fact, ACLF is a dynamic process in which the variables at the time of hospitalization are predicted to vary over time, so the clinical processes and outcomes change accordingly [21-23]. Therefore, the change of ALB level between stem cell therapy and control group may be better for the assessment of liver function improvement than the assessment based on 


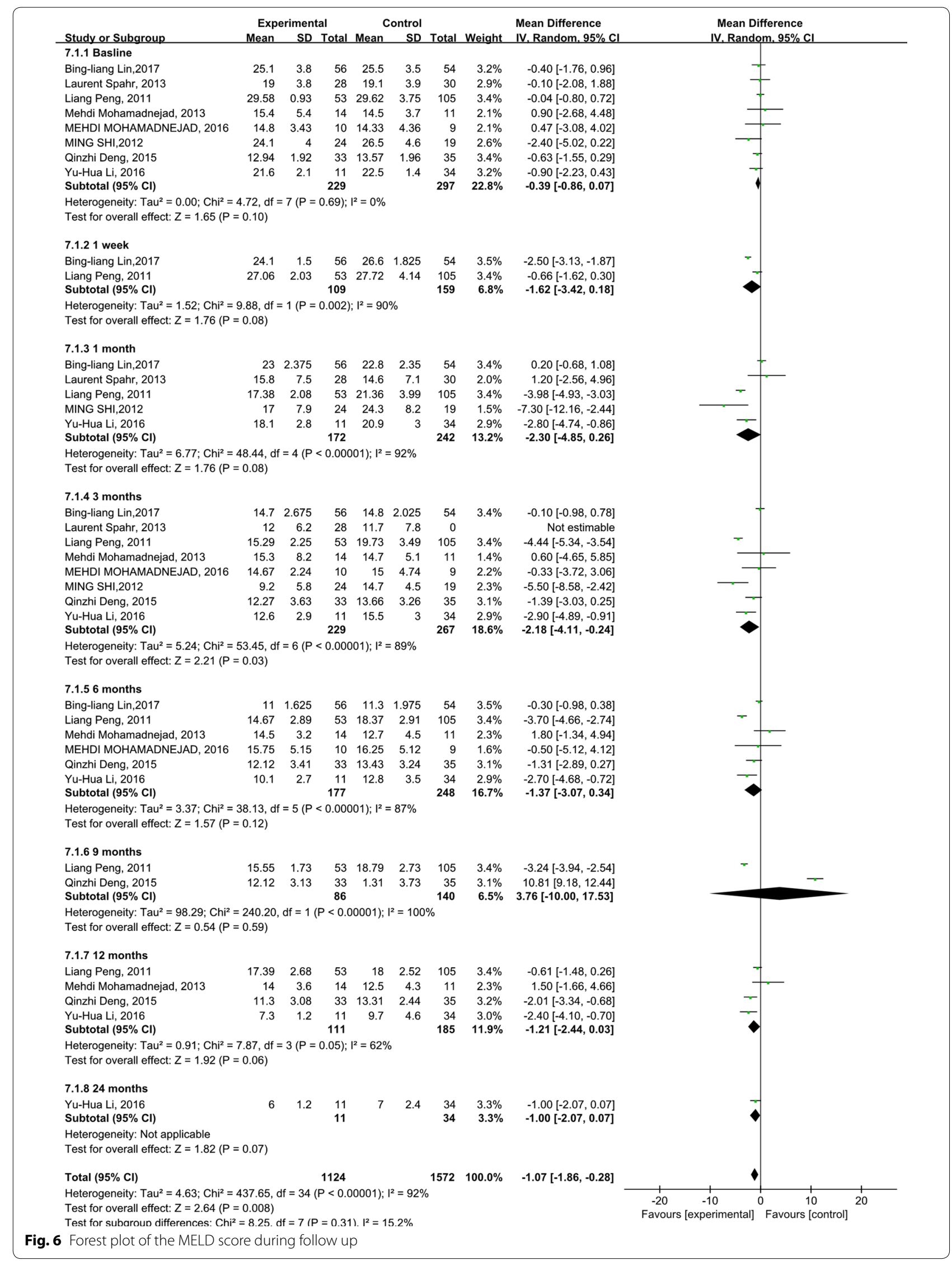




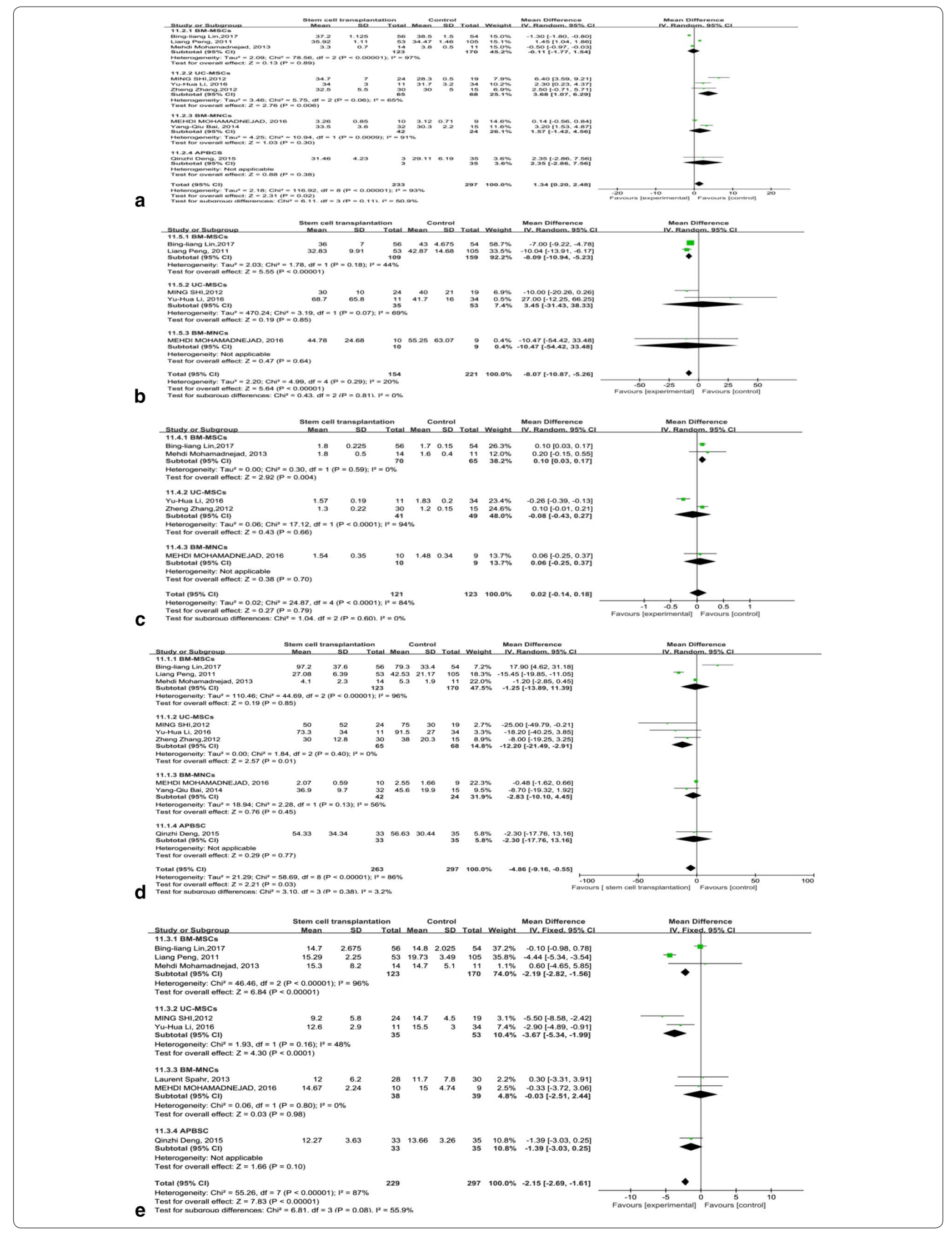


(See figure on previous page.)

Fig. 7 Futher subgroup analysis for 3-month clinical performance according to the stem cell types. a Forest plot of the ALB during 3 months. b Forest plot of the ALT during 3 months. c Forest plot of the INR during 3 months. $\mathbf{d}$ Forest plot of the TBIL during 3 months. e Forest plot of MELD scores during 3 months

static baseline variables. However, there were not enough $\triangle \mathrm{ALB}$ data for this systematic review and meta-analysis now.

The INR levels did not show any obvious change compared with the control groups $(\mathrm{p}=0.64)$. The reason may be that patients received plasma after admission. However, heterogeneity was high in these studies, although subgroup analysis was done $\left(\mathrm{I}^{2}=82-95 \%\right)$. More RCTs are urgently needed.

Stem cell therapy significantly decreased the TBIL levels at $1,3,12$ months $(\mathrm{p}=0.0008 ; \mathrm{p}=0.04$; $\mathrm{p}=0.007)$. The ALT level in the stem cell therapy group also gradually decreased and was lower than baseline and the corresponding controls at the 1 week, 1 month and 6 months time points. However, there were no significant differences in ALT level at 9, 12 and 24 months after stem cell therapy. Meanwhile, no significant difference was found in TBIL level compared with the control group at 9 and 24 months time points. It is indicated that stem cell therapy can alleviate liver damage in patients with ACLF in the short-term, but the longterm outcome for ACLF patients is not remarkable.

The MELD scores have been used to predict mortality of cirrhosis patients awaiting liver therapy, as well as prioritize liver allocation [24-27]. In this study, a significant difference can be found between the stem cell therapy and control groups $(p=0.008)$. It is suggested that stem cell therapy can reduce the MELD score and improve the prognosis of ACLF patients, so as to relieve the social burden for lack of liver donors.

There are some limitations in this study. We pooled different stem cell types in the publications, leading to a high heterogeneity degree. However, it was impossible to conduct all subgroup analyses according to the stem cell type. Because there were no enough data for us to do the subgroup analysis. Thus, we only selected 3 months for the subgroup analysis. It seems that the subgroup analysis can better reduce the heterogeneity in some degree. Compared with other types of stem cells, UC-MSCs had a better effect on increasing the ALB $(\mathrm{p}=0.006)$. After the subgroup analyses on the effects of stem cell type at 3 months of followup for ALT and INR, BM-MSCs can obviously reduce the ALT level and INR, which is better than other stem cell types $(\mathrm{p}<0.00001 ; \mathrm{p}=0.004)$. As for TBIL, it seems that UC-MSCs had a better effect on reducing the TBIL than BM-MNCs and BM-MSCs $(p=0.01)$. It is suggested that UC-MSCs and BM-MNCs can significantly decrease the MELD score, which is better than BM-MNCs at 3 months of follow-up ( $<<0.00001$; $\mathrm{p}<0.0001$ ). Above all, in our study, MSCs may be better than BM-MNCs in the stem cells transplantation of ACLF. However, more clinical trials with different stem cell types are needed to verify the clinical outcomes.

\section{Conclusions}

This study suggests that the clinical outcomes of stem cell therapy were satisfactory in patients with ACLF in the short-term. Meanwhile, MSCs may be better than BM-MNCs in the stem cells transplantation of ACLF. Nevertheless, more attention should focus on large multicenter clinical trials and further basic research. Moreover, well-designed RCTs are needed to verify the clinical effectiveness of the long-term outcomes.

\section{Abbreviations}

ACLF: acute-on-chronic liver failure; LT: liver transplantation; ALB: albumin; INR: international normalized ratio; MELD: model for end-stage liver disease score; ALT: alanine aminotransferase; TBIL: total bilirubin; MSCs: mesenchymal stem cells; HSCs: hematopoietic stem cells; BMCs: bone marrow cells; PRISMA: preferred reporting items for systematic reviews and meta-analyses; SMTs: standards medical treatments; MD: mean difference; Cl: confidential interval; CLD: chronic liver disease; RCTs: random controlled trials.

\section{Authors' contributions}

RX wrote the manuscript paper. QM designed the study. JL, JD, YZ, QY and HY collected data, performed some analysis. All authors read and approved the final manuscript.

\section{Acknowledgements}

Not applicable.

Competing interests

The authors declare that they have no competing interests.

Availability of data and materials

Not applicable.

Consent for publication

Not applicable.

Ethics approval and consent to participate Not applicable.

\section{Funding}

The study was supported by the National Natural Science Foundation of China (No. 81470877), and Capital Health Research and Development of Special (2011-2018-09). 


\section{Publisher's Note}

Springer Nature remains neutral with regard to jurisdictional claims in published maps and institutional affiliations.

Received: 20 January 2018 Accepted: 27 March 2018

Published online: 10 May 2018

\section{References}

1. Bernal W, Jalan R, Quaglia A, Simpson K, Wendon J, Burroughs A. Acuteon-chronic liver failure. Lancet. 2015;10003:1576-87.

2. Liu Q, Liu Z, Wang T, Wang Q, Shi X, Dao W. Characteristics of acute and sub-acute liver failure in China: nomination, classification and interval. J Gastroenterol Hepatol. 2007;12:2101-6.

3. Sarin SK, Kumar A, Almeida JA, et al. Acute-on-chronic liver failure: consensus recommendations of the Asian Pacifc association for the study of the liver (APASL). Hepatol Int. 2009;3(1):269-82.

4. Asrani SK, Simonetto DA, Kamath PS. Acute-on-chronic liver failure. Clin Gastroenterol Hepatol. 2015;13(12):2128-39.

5. Peng L, Xie DY, Lin BL, et al. Autologous bone marrow mesenchymal stem cell therapy in liver failure patients caused by hepatitis B: short-term and long-term outcomes. Hepatology. 2011;54(3):820-8.

6. Mohamadnejad M, Alimoghaddam K, Bagheri M, et al. Randomized placebo-controlled trial of mesenchymal stem cell therapy in decompensated cirrhosis. Liver Int. 2013:33:1490-6.

7. Spahr L, Chalandon Y, Terraz S, et al. Autologous bone marrow mononuclear cell therapy in patients with decompensated alcoholic liver disease: a randomized controlled trial. PLOS ONE. 2013;8:e53719.

8. Bai YQ, Yang YX, Yang YG, et al. Outcomes of autologous bone marrow mononuclear cell therapy in decompensated liver cirrhosis. World J Gastroenterol. 2014;20:8660-6.

9. Terai S, Sakaida I, Yamamoto N, et al. An in vivo model for monitoring trans-differentiation of bone marrow cells into functional hepatocytes. J Biochem. 2003;134:551-8.

10. Peng L, Li H, Gu L, Peng XM, Huang YS, Gao ZL. Comparison of biological characteristics of marrow mesenchymal stem cells in hepatitis B patients and normal adults. World J Gastroenterol. 2007;13(11):1743-6.

11. Zeng $X$, Zhang Y, Kwong JS, et al. The methodological quality assessment tools for preclinical and clinical studies, systematic review and metaanalysis, and clinical practice guideline: a systematic review. J Evid Based Med. 2015;8(1):2-10.

12. Guyatt G, Oxman AD, Akl EA, et al. GRADE guidelines: 1. IntroductionGRADE evidence profiles and summary of findings tables. J Clin Epidemiol. 2011;64(4):383-94.

13. Hozo SP, Djulbegovic B, Hozo I. Estimating the mean and variance from the median, range, and the size of a sample. BMC Med Res Methodol. 2005:5:13.
14. Zheng MH, Wu SJ, Shi KQ, et al. Conditional survival estimate of acuteon-chronic hepatitis B liver failure: a dynamic prediction based on a multicenter cohort. Oncotarget. 2015;6(27):23261-71.

15. Connick P, Kolappan M, Crawley C, et al. Autologous mesenchymal stem cells for the treatment of secondary progressive multiple sclerosis: an open label phase 2a proof-of-concept study. Lancet Neurol. 2012;11(2):150-6.

16. Jiang R, Han Z, Zhuo G, et al. Therapy of placenta-derived mesenchymal stem cells in type 2 diabetes: a pilot study. Front Med. 2011:5(1):94-100

17. Makkar RR, Smith RR, Cheng K, et al. Intracoronary cardiosphere-derived cells for heart regeneration after myocardial infarction (CADUCEUS): a prospective, randomised phase 1 trial. Lancet. 2012;379(9819):895-904.

18. De Miguel MP, Fuentes-Julián S, Blázquez-Martínez A, et al. Immunosuppressive properties of mesenchymal stem cells: advances and applications. Curr Mol Med. 2012;12(5):574-91.

19. Gao F, Chiu SM, Motan DA, et al. Mesenchymal stem cells and immunomodulation: current status and future prospects. Cell Death Dis. 2016;7:e2062.

20. Kaundal U, Bagai U, Rakha A. Immunomodulatory plasticity of mesenchymal stem cells: a potential key to successful solid organ transplantation. J Transl Med. 2018;16(1):31.

21. Huo TI, Wu JC, Lin HC, et al. Evaluation of the increase in model for end-stage liver disease (DeltaMELD) score over time as a prognostic predictor in patients with advanced cirrhosis: risk factor analysis and comparison with initial MELD and Child-Turcotte-Pugh score. J Hepatol. 2005;42:826-32.

22. Shi $M$, Zhang Z, Xu R, et al. Human mesenchymal stem cell transfusion is safe and improves liver function in acute-on-chronic liver failure patients. Stem Cells Transl Med. 2012;1(10):725-31.

23. Zhang Z, Lin H, Shi M, et al. Human umbilical cord mesenchymal stem cells improve liver function and ascites in decompensated liver cirrhosis patients. J Gastroenterol Hepatol. 2012;27(s2):112.

24. Bai $Y Q$, Yang $Y X$, Yang $Y G$, et al. Outcomes of autologous bone marrow mononuclear cell transplantation in decompensated liver cirrhosis. World J Gastroenterol. 2014;20(26):8660-6.

25. Deng Q, Cai T, Zhang S, et al. Autologous peripheral blood stem cell transplantation improves portal hemodynamics in patients with hepatitis B virus-related decompensated cirrhosis. Hepat Mon. 2015;15(12):e32498.

26. Mohamadnejad M, Vosough M, Moossavi S, et al. Intraportal infusion of bone marrow mononuclear or CD133+ cells in patients with decompensated cirrhosis: a double-blind randomized controlled trial. Stem Cells Transl Med. 2015:5(1):87-94.

27. Lin BL, Chen JF, Qiu WH, et al. Allogeneic bone marrow-derived mesenchymal stromal cells for hepatitis B virus-related acute-on-chronic liver failure: a randomized controlled trial. Hepatology. 2017;66(1):209-19.
Ready to submit your research? Choose BMC and benefit from:

- fast, convenient online submission

- thorough peer review by experienced researchers in your field

- rapid publication on acceptance

- support for research data, including large and complex data types

- gold Open Access which fosters wider collaboration and increased citations

- maximum visibility for your research: over 100M website views per year

At BMC, research is always in progress.

Learn more biomedcentral.com/submissions 